\title{
The role of E-box-, G-box- and RY-motif-binding proteins in regulation of ethylene response in Arabidopsis thaliana
}

\author{
Pukhovaya E. ${ }^{1,2 *}$, Levitsky V. ${ }^{1,2}$, Oshchepkov D. ${ }^{1}$, Zemlyanskaya E. ${ }^{1,2}$ \\ ${ }^{1}$ Institute of Cytology and Genetics, SB RAS, Novosibirsk, Russia \\ ${ }^{2}$ Novosibirsk State University, Novosibirsk, Russia \\ *e-mail: e.pukhovaya@gmail.com
}

Plant hormone ethylene is a major regulator of growth and development that plays an important role in various processes such as seed germination, response to biotic and abiotic stresses, senescence etc. Ethylene signaling proceeds via a linear pathway that activates EIN3 and EIL1 transcription factors (TF) - the key regulators of ethylene response. Besides EIN3 binding to gene promoters, co-factors are often required to trigger gene expression upon ethylene treatment. In this work, we perform a genomewide analysis of EIN3 binding to investigate complex mechanisms of EIN3-mediated gene expression upon ethylene treatment. De novo motif search in EIN3 ChIP-seq peaks (publicly available data) with Homer tool found a variety of enriched motifs. Besides EBS-like motif (well-known EIN3-binding site) $(p<1 \mathrm{e}-109)$, E-box-like motif $(p<1 \mathrm{e}-125)$, G-box-like motif $(p<1 \mathrm{e}-47)$, and RY-like motif $(p<1 \mathrm{e}-52)$ were in the top. Intriguingly, E-box-like motif rather than EBS-like motif was the first ranked. Using Tomtom tool, E-box-, G-box- and RY-like motifs were annotated as the binding sites for bZIP family group I TFs, PIF family TFs and FUS3 binding sites, correspondingly. Enrichment of the motifs annotated as the binding sites of EIN3-unrelated TFs supports indirectly a possible role of these TFs as EIN3 co-factors. To further investigate this possibility, we performed an analysis of EIN3 and bZIP29/PIF4/FUS3 binding cooccurrence in gene promoters and found it statistically significant $(p<2.2 \mathrm{e}-16)$. It is noteworthy that FUS3, PIF family and bZIP family group I TFs are known to take part in several ethylene-regulated processes. Therefore, according to our results we suggest that FUS3, PIF family and bZIP family group I TFs could influence EIN3 functioning to regulate gene expression on the genome-wide level. These TFs are promising candidates for future investigations of EIN3 functional partners. 